\title{
Port-based Modeling of Human-Robot Collaboration towards Safety-enhancing Energy Shaping Control
}

\author{
Milad Geravand Erfan Shahriari
}

\begin{abstract}
While collision detection and contact-related injury reduction in physical human-robot interaction has been studied intensively, safety issues in physical human-robot collaboration (pHRC) with continuous coupling of human and $\operatorname{robot}(\mathrm{s})$ has received little attention so far. We develop an energy monitoring control system that observes energy flows among the different subsystems involved in pHRC, shaping them to improve human safety according to selected metrics. PortHamiltonian formalisms are used to model each sub-system and their interconnection. An energy-based compliance controller that enhances safety by adapting the robot behavior is proposed and validated through extensive simulations.
\end{abstract}

\section{INTRODUCTION}

Robotics research is progressing towards physical interaction between humans and robots, with multiple potential applications in industry (robot co-workers) as well as in rehabilitation and assistive robotics, e.g., robotic devices that provide support to patients and elderlies during walking or transfers. Close interaction of a human with robots requires proper hardware and software components so as to enhance safety of the user. Intrinsically safe mechanisms as well as lightweight and compliant designs have been proven to increase robot safety [1]-[4]. Nowadays, a variety of motion planning and reactive control methods exists that are able to prevent human-robot collisions, using sensors to monitor the workspace, and to reduce contact-related injuries, see e.g. [5]-[10].

In many tasks, however, there is a need of establishing intentional, continuous, and often multiple contacts between human and $\operatorname{robot}(\mathrm{s})$. Such a physical Human-Robot Collaboration (pHRC) comes with a series of new challenges for modeling the overall dynamical system and for the design of safe robot control. A human and one or more robots may in fact interact directly or via a carried object, at a single point or over several interaction ports, with multiple, changing and intermittent contact situations, resulting thus in a very complex dynamical system which is difficult to model. In addition, safety-related control issues have to be

This work is supported in part by the MOBOT project within the 7 th Framework Programme of the European Union, under the grand agreement n. 600796 and the Institute of Advanced Studies of the Technische Universität München.

M. Geravand is with Chair of Automatic Control Engineering, Technische Universität München, Munich, Germany, (milad.geravand@tum.de). E. Shahriari is with Robotics Innovation Center, Deutsches Forschungszentrum für Künstliche Intelligenz DFKI GmbH, Bremen, Germany, (erfan.shahriariedfki.de). A. De Luca is with Dipartimento di Ingegneria Informatica, Automatica e Gestionale, Sapienza Università di Roma, Rome, Italy, (deluca@diag.uniroma1.it). A. Peer is with Bristol Robotics Laboratory, University of the West of England, Bristol, UK, (Angelika.Peer@brl.ac.uk).

\author{
Alessandro De Luca Angelika Peer
}

redefined for continuous pHRC since behaviors such as collision avoidance and reactive escape control [6] are no longer representative ones.

Since pHRC can be seen as an exchange of force and motion signals over contact points, an energy and port-based approach provides a very powerful tool for both modeling and control design. While several researchers have already used the concept of energy and power for robot collision detection [8] and safe reaction control [11], a systematic energy-based modeling and control approach to pHRC tasks involving continuous contact and a varying number and location of contact points is missing so far. The Port-Hamiltonian $(\mathrm{PH})$ formalism, which is a domain-independent concept, has proven to be very successful for modeling complex systems [12], [13]. It provides a framework to describe a system in terms of energy variables and interconnection of sub-systems by means of power ports. So far, it was adopted to model the dynamical behavior of robots with rigid or flexible links [14], hybrid hopping robots [15], underactuated aerial vehicles [16], soft finger manipulation [17], as well as for the control of bipedal walking robots [18].

This paper introduces a very general PH model for the collaborative task of manipulating a rigid object by a humanrobot team. Each sub-system is modeled independently using the power port concept, and then interconnected to form the overall system. This allows to include without essential model changes also transition events from contact to noncontact and vice versa, or changes in the overall system dynamics, e.g., when adding a further collaborative robot to the picture. Based on this model, an energy monitoring system is defined that continuously observes the energy flows between all components, but especially over the interaction port with the human. Finally, a safety-enhancing controller is proposed that shapes the energy exchanged with the human whenever a harmful energy flow or human fatigue is observed.

\section{BACKGROUND}

This section provides a brief overview of the basic components of the port-based modeling framework including the Dirac structure, the Port-Hamiltonian formalism and screw theory. The reader is referred to [19]-[21] for details.

\section{A. Port-based modeling framework}

Energy is the underlying concept for the port-based modeling. Each sub-system interacts with the others through the rate of change of energy, namely power, as a dual product of the two power-conjugate port variables, flow $f$ and effort 
$e$. The interconnection between sub-systems is described by a network topology called Dirac structure $\mathcal{D}$, which mathematically represents how the power flows among the ports of the structure. With $\mathcal{F}$ being the linear space of flows $(f \in \mathcal{F})$ and $\mathcal{F}^{*}$ the dual linear space of efforts $(e \in \mathcal{E})$, the Dirac structure is expressed in the space $\mathcal{F} \times \mathcal{F}^{*}$ as

$$
\mathcal{D}=\left\{(f, e) \in \mathcal{F} \times \mathcal{F}^{*} \mid F f+E e=0\right\}
$$

with $F$ and $E$ two specific mappings imposing the powerconservation in the whole system.

Elements in the network are characterized by their energetic behavior and are grouped into energy storage ports $\left(f_{S}, e_{S}\right)$, resistive ports $\left(f_{R}, e_{R}\right)$ for energy dissipation, control ports $\left(f_{C}, e_{C}\right)$ and interaction ports $\left(f_{I}, e_{I}\right)$.

\section{B. PH formulation}

The PH formulation represents the input/output relation of a port-based model. Considering the Hamiltonian function $H$ of the total system energy, the standard representation of a PH system is given by:

$$
\left\{\begin{array}{l}
\dot{\boldsymbol{x}}=[\boldsymbol{J}(\boldsymbol{x})-\boldsymbol{R}(\boldsymbol{x})] \frac{\partial H}{\partial \boldsymbol{x}}+\boldsymbol{G}(\boldsymbol{x}) \boldsymbol{u} \\
\boldsymbol{y}=\boldsymbol{G}^{T}(\boldsymbol{x}) \frac{\partial H}{\partial \boldsymbol{x}}
\end{array}\right.
$$

where $\boldsymbol{J}(\boldsymbol{x})$ is a skew symmetric matrix, $\boldsymbol{R}(\boldsymbol{x}) \geq 0$ is the symmetric dissipation matrix, $\boldsymbol{G}(\boldsymbol{x})$ is a mapping matrix, $\boldsymbol{x}$ is the state associated to the storage elements and $\boldsymbol{u}, \boldsymbol{y}$ are the input and output variables, respectively.

A system presented in PH form (2) can be easily represented by its underlying Dirac structure:

$$
\left[\begin{array}{c}
-\dot{\boldsymbol{x}} \\
\boldsymbol{e}_{R} \\
\boldsymbol{y}
\end{array}\right]=\left[\begin{array}{ccc}
-\boldsymbol{J}(\boldsymbol{x}) & -\boldsymbol{G}_{R}(\boldsymbol{x}) & -\boldsymbol{G}(\boldsymbol{x}) \\
\boldsymbol{G}_{R}^{T}(\boldsymbol{x}) & 0 & 0 \\
\boldsymbol{G}^{T}(\boldsymbol{x}) & 0 & 0
\end{array}\right]\left[\begin{array}{c}
\frac{\partial H}{\partial \boldsymbol{x}} \\
\boldsymbol{f}_{R} \\
\boldsymbol{u}
\end{array}\right]
$$

with $\boldsymbol{R}(\boldsymbol{x})=\boldsymbol{G}_{R}(\boldsymbol{x}) \boldsymbol{Y}_{R} \boldsymbol{G}_{R}^{T}(\boldsymbol{x})$ for a linear admittance relation $\boldsymbol{f}_{R}=-\boldsymbol{Y}_{R} \boldsymbol{e}_{R}$.

\section{Twists and wrenches}

In physical systems, a twist is the relative instantaneous motion of a body with frame $\Psi_{i}$ with respect to a body with frame $\Psi_{j}$ expressed in frame $\Psi_{0}$ and is mathematically given by ${ }^{0} \boldsymbol{T}_{i}^{j}=[\boldsymbol{\omega}, \boldsymbol{v}]^{T}$ where $\boldsymbol{\omega}$ and $\boldsymbol{v}$ are the angular and translational velocities. The coordinate transformation of a twist is defined by ${ }^{0} \boldsymbol{T}_{i}^{j}=\boldsymbol{A}_{l}^{0 l} \boldsymbol{T}_{i}^{j}$ in which

$$
\boldsymbol{A}_{l}^{0}=\left[\begin{array}{cc}
\boldsymbol{R}_{l}^{0} & 0 \\
\boldsymbol{p}_{l}^{0^{*}} \boldsymbol{R}_{l}^{0} & \boldsymbol{R}_{l}^{0}
\end{array}\right]
$$

$\boldsymbol{R}_{l}^{0}$ the rotation matrix, and $\boldsymbol{p}_{l}^{0^{*}}$ the skew-symmetric matrix form for the displacement between frames $\Psi_{0}$ and $\Psi_{l}$.

The wrench applied to the body with frame $\Psi_{i}$ acting on frame $\Psi_{j}$ is an element of the dual space to the twist vector space and is represented as ${ }^{0} \boldsymbol{W}_{i}^{j}=[\boldsymbol{m}, \boldsymbol{f}]^{T}$ with torques $\boldsymbol{m}$ and forces $\boldsymbol{f}$. The coordinate transformation of a wrench is then defined as ${ }^{0} \boldsymbol{W}_{i}^{j}=\boldsymbol{A}_{l}^{0^{T}}{ }^{l} \boldsymbol{W}_{i}^{j}$.

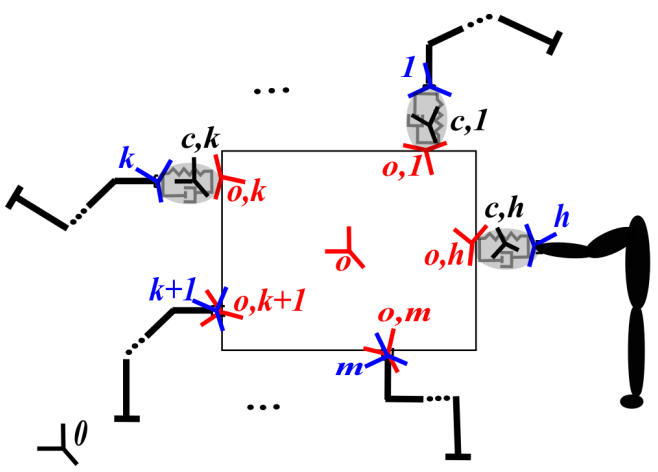

Fig. 1. An example scenario of object transportation by a human and $m$ collaborative robots and possible associated frame definition.

\section{PH Modeling OF HRC}

In this section, we present an energy and port-based modeling approach for the collaborative task of jointly manipulating a rigid bulky object by a human-robot team, see a general example in Fig. 1. We believe that the selected scenario is representative for modeling different HRC scenarios, which include physical contact between different sub-systems. The mathematical representation of each sub-system including robot(s), human as well as contacts (e.g. between human and object or object and robot) are introduced first, then the whole system is described by the interconnection of the different sub-systems through their interaction ports. Please note that due to space restrictions, for each sub-system we present only its underlying Dirac structure while their transformation to $\mathrm{PH}$ formulation is very straightforward, and is thus not reported explicitly. The overall system is finally presented in $\mathrm{PH}$ form, which is more convenient for the control of the system.

\section{A. PH modeling of the robot}

For each robot $r$ among $m$ collaborative robots, $r \in$ $\{1,2, \ldots, m\}$, having $n_{r}$ degrees of freedom (DOF), generalized coordinates $\mathbf{q}_{r}=\left(q_{1}, \ldots, q_{n_{r}}\right)^{T}$, and symmetric, positive definite inertia matrix $\boldsymbol{M}_{r}\left(\boldsymbol{q}_{r}\right)$, the energy ports for storing potential and kinetic energies, the control ports, as well as the interaction ports have to be defined.

Considering the robot $r$, the Hamiltonian function and state variables for the storage port describing the potential energy $\left\langle\dot{\boldsymbol{q}}_{r}, \frac{\partial H_{r}}{\partial \boldsymbol{q}_{r}}\right\rangle$ are defined based on the robot gravitational energy $U_{r}\left(\boldsymbol{q}_{r}\right)$ and the robot configuration vector $\boldsymbol{q}_{r}$. The Hamiltonian function and state variables for the storage port describing the kinetic energy are given by $\frac{1}{2} \boldsymbol{p}_{r}^{T} \boldsymbol{M}_{r}^{-1}\left(\boldsymbol{q}_{r}\right) \boldsymbol{p}_{r}$ and are defined with the help of the vector of generalized momenta $\boldsymbol{p}_{r}=\left(p_{1}, \ldots, p_{n_{r}}\right)^{T}$, where $\boldsymbol{p}_{r}=\boldsymbol{M}_{r}\left(\boldsymbol{q}_{r}\right) \dot{\boldsymbol{q}}_{r}$.

The resistive port $\left\langle\boldsymbol{e}_{R, r}, \boldsymbol{f}_{R, r}\right\rangle$ describes the dissipative behavior of the robot, e.g. due to friction in joints or transmissions. The port variables are related by $\boldsymbol{f}_{R, r}=-\boldsymbol{D}_{r} \boldsymbol{e}_{R, r}$ where $\boldsymbol{f}_{R, r}$ means the flow variable representing the dissipative joint torques, $\boldsymbol{e}_{R, r}$ the effort variable representing the joint velocities and $\boldsymbol{D}_{r}$ the dissipation matrix.

Finally, the control $\left\langle\dot{\boldsymbol{q}}_{r}, \boldsymbol{\tau}_{r}\right\rangle$ and interaction ports $\left\langle{ }^{0} \boldsymbol{T}_{r}^{0},{ }^{0} \boldsymbol{W}_{o, r}^{r}\right\rangle$ describe the robot behavior with respect to 
its actuation as well as the interaction with the environment. In the considered scenario this means the robot interaction with the object. The flow variable ${ }^{0} \boldsymbol{T}_{r}^{0}$ thus, describes the instantaneous motion of the robot end-effector, while the effort variable ${ }^{0} \boldsymbol{W}_{o, r}^{r}$ describes the interaction forces and torques that can be measured between object and end-effector of the robot $r$ at their contact point. For the sake of simplicity both flow and effort variables for each robot are expressed in the world reference frame, see Fig. 1.

The total Hamiltonian of the robot is then given by

$$
H_{r}=\frac{1}{2} \boldsymbol{p}_{r}^{T} \boldsymbol{M}_{r}^{-1}\left(\boldsymbol{q}_{r}\right) \boldsymbol{p}_{r}+U_{r}\left(\boldsymbol{q}_{r}\right)
$$

while the underlying Dirac structure of the robot can be formulated following the Hamiltonian principle (see [19])

$$
\begin{aligned}
{\left[\begin{array}{c}
\dot{\boldsymbol{p}}_{r} \\
\dot{\boldsymbol{q}}_{r} \\
\boldsymbol{e}_{R, r} \\
{ }^{0} \boldsymbol{T}_{r}^{0} \\
\dot{\boldsymbol{q}}_{r}^{0}
\end{array}\right] } & =\left[\begin{array}{cc}
\mathbf{0}_{n_{r} \times n_{r}} & -\boldsymbol{N}_{r} \\
\boldsymbol{N}_{r}^{T} & \mathbf{0}_{\left(3 n_{r}+6\right) \times\left(3 n_{r}+6\right)}
\end{array}\right]\left[\begin{array}{c}
\frac{\partial H_{r}}{\partial \boldsymbol{p}_{r}} \\
\frac{\partial H_{r}}{\partial \boldsymbol{q}_{r}} \\
-\boldsymbol{f}_{R, r} \\
-{ }^{0} \boldsymbol{W}_{o, r}^{r} \\
-\boldsymbol{\tau}_{r}
\end{array}\right] \\
\boldsymbol{N}_{r} & =\left[\boldsymbol{I}_{n_{r}}, \boldsymbol{I}_{n_{r}}, \boldsymbol{J}_{r}^{T}\left(\boldsymbol{q}_{r}\right), \boldsymbol{I}_{n_{r}}\right]
\end{aligned}
$$

with robot Jacobian $\boldsymbol{J}_{r}$ and identity matrix $\boldsymbol{I}_{n_{r}}$ of order $n_{r}$.

\section{B. PH modeling of the object}

The dynamics of the object with total mass $M_{o}$ and body inertia matrix $\boldsymbol{I}_{O}$ is described by interaction ports and storing ports taking into account the potential energy and linear and angular kinetic energy.

The number of interaction ports depends on the number of contacts between the object and the human or collaborative $\operatorname{robot}(\mathrm{s})$. Each interaction port includes the flow variable ${ }^{0} \boldsymbol{T}_{o, t}^{0}$, which describes the instantaneous motion of the object at the contact point with element $t$, either with the human or with the robots $t \in\{h, 1, \cdots, m\}$, and the effort variable ${ }^{0} \boldsymbol{W}_{t}^{o, t}$, which describes the wrenches applied by the element $t$ to the object at the specific contact point, both expressed in frame 0 . Given the position vector ${ }^{o} \boldsymbol{P}_{o, t}$ of each contact point between the object and the element $t$ expressed in the object frame, the input matrix $\boldsymbol{G}_{t}$ for each interaction port can be written as follows

$$
\boldsymbol{G}_{t}=\left[\begin{array}{cc}
\boldsymbol{I}_{3} & \mathbf{0}_{3 \times 3} \\
\left({ }^{o} \boldsymbol{P}_{o, t}\right)^{*} & \boldsymbol{I}_{3}
\end{array}\right]^{T}
$$

with identity matrix $\boldsymbol{I}_{3}$, and $3 \times 3$ skew-symmetric matrix $\left({ }^{o} \boldsymbol{P}_{o, t}\right)^{*}$ associated to the displacement vector ${ }^{o} \boldsymbol{P}_{o, t}$.

The position $\boldsymbol{x}_{o}$ of the center of mass of the object is the state variable for the storage port $\left\langle\dot{\boldsymbol{x}}_{o}, \frac{\partial H_{o}}{\partial \boldsymbol{x}_{o}}\right\rangle$ describing the gravitational energy with the Hamiltonian function $H_{o}=$ $M_{o} \boldsymbol{g}^{T} \boldsymbol{x}_{o}$ and $\boldsymbol{g}=\left[0,-g_{0}, 0\right]^{T}$ the gravity vector.

The storage ports for the linear kinetic energy $\left\langle\dot{\boldsymbol{p}}_{o}, \frac{\partial H}{\partial \boldsymbol{p}_{0}}\right\rangle$ and angular kinetic energy $\left\langle\dot{\boldsymbol{l}}_{o}, \frac{\partial H}{\partial \boldsymbol{l}_{o}}\right\rangle$ are characterized by their state variables $\boldsymbol{p}_{o}=M_{o} \boldsymbol{v}_{o}$ for the object's linear momentum and $\boldsymbol{l}_{o}=\boldsymbol{I}_{o} \boldsymbol{\omega}_{o}$ for the object's angular momentum. Corresponding Hamiltonian functions for aforementioned storage ports are presented as $\frac{1}{2} \boldsymbol{p}_{o}^{T} M_{o}^{-1} \boldsymbol{p}_{o}$ and $\frac{1}{2} \boldsymbol{l}_{o}^{T} \boldsymbol{I}_{o}^{-1} \boldsymbol{l}_{o}$. In above formulas, $\boldsymbol{v}_{o}$ is the linear velocity of the object, $\boldsymbol{\omega}_{o}$ the angular velocity, and $\boldsymbol{I}_{o}$ the inertia tensor of the object. Please note that assigning two storage ports is performed to be able to explicitly analyse linear and angular motions of the object independently, although these two ports could be also combined into one as done e.g. in Section III-A.

The total Hamiltonian function of the object is given by

$$
H=\frac{1}{2} \boldsymbol{p}_{o}^{T} M_{o}^{-1} \boldsymbol{p}_{o}+\frac{1}{2} \boldsymbol{l}_{o}^{T} \boldsymbol{I}_{o}^{-1} \boldsymbol{l}_{o}+\left(-M_{o} \boldsymbol{g}^{T} \boldsymbol{x}_{\boldsymbol{o}}\right) .
$$

Following the Hamiltonian principle this results in the following formulation of the corresponding Dirac structure

$$
\begin{gathered}
{\left[\begin{array}{c}
{\left[\begin{array}{c}
\dot{\boldsymbol{p}}_{o} \\
\dot{\boldsymbol{l}}_{o}
\end{array}\right]} \\
\dot{\boldsymbol{x}}_{o} \\
{ }^{0} \boldsymbol{T}_{o, 1}^{0} \\
\vdots \\
{ }^{0} \boldsymbol{T}_{o, m}^{0} \\
{ }^{0} \boldsymbol{T}_{o, h}^{0}
\end{array}\right]=\left[\begin{array}{cc}
\mathbf{0}_{6 \times 6} & -\boldsymbol{N}_{o} \\
\boldsymbol{N}_{o}^{T} & \mathbf{0}_{(6 m+9) \times(6 m+9)}
\end{array}\right]\left[\begin{array}{c}
{\left[\begin{array}{c}
\frac{\partial H}{\partial \boldsymbol{p}_{o}} \\
\frac{\partial H}{\partial \boldsymbol{l}_{o}}
\end{array}\right]} \\
\frac{\partial H}{\partial \boldsymbol{x}_{o}} \\
-{ }^{0} \boldsymbol{W}_{1}^{o, 1} \\
\vdots \\
-{ }^{o} \boldsymbol{W}_{m}^{o, m} \\
-{ }^{o} \boldsymbol{W}_{h}^{o, h}
\end{array}\right]} \\
\boldsymbol{N}_{o}=\left[\boldsymbol{I}_{3}, \mathbf{0}_{3 \times 3}, \boldsymbol{G}_{1}, \cdots, \boldsymbol{G}_{m}, \boldsymbol{G}_{h}\right]
\end{gathered}
$$

\section{PH modeling of the human}

Since the number of degrees of freedom does not affect the procedure of modeling based on the $\mathrm{PH}$ formalism, without loss of generality we consider a $n_{h}$ DOF human arm structure, which can e.g. include flexion-extension movements in the shoulder, elbow and wrist. The visco-elasticity of the human skin (or even arm) can also be considered within the compliant contact model as detailed in Section III-D.

Similar to section III-A, an energy storage port corresponding to the arm's potential energy, an interaction port representing the dynamical behavior due to the contact between human and object, and a control port for the joint torques generated by human muscles are considered. The final $\mathrm{PH}$ formulation results in the same structure as (2).

\section{Physical contacts}

The physical contact between human, object and robot(s) are established using rigid or compliant contacts. Thus, two physical contact models are formulated using port variables.

1) Rigid contact: In this case, the physics of contact is described by a kinematic constraint imposed on the relative motion of two connected sub-systems $i$ and $j$, which imposes the relative twist ${ }^{b} \boldsymbol{T}_{i}^{j}$ to belong to a specific subspace of se(3), which includes only feasible directions of motion.

Thus, kinematic constraints of zero relative twist between component $i$ and component $j$ are introduced by setting ${ }^{0} \boldsymbol{T}_{i}^{j}={ }^{0} \boldsymbol{T}_{i}^{0}-{ }^{0} \boldsymbol{T}_{j}^{0}=\mathbf{0}_{6}$. Zero rate of change of ${ }^{0} \boldsymbol{T}_{i}^{j}$ is used to compute the imposed wrenches ${ }^{0} \boldsymbol{W}_{j}^{i}$ and ${ }^{0} \boldsymbol{W}_{i}^{j}$, which are a consequence of the kinematic constraints ${ }^{1}$.

\footnotetext{
${ }^{1}$ Please note that ${ }^{0} \boldsymbol{W}_{j}^{i}=-{ }^{0} \boldsymbol{W}_{i}^{j}$ due to the power-conserving nature of the connection.
} 
2) Compliant contact: Compliant contacts are modeled as a coupling of an elastic and a dissipative element [22], [23]. Elasticity in the contact is modeled by a storage port with state variable $s$ and Hamiltonian function $H_{s}=\frac{1}{2} \boldsymbol{s}^{T} \boldsymbol{K}_{s} \boldsymbol{s}$, with $\boldsymbol{K}_{s}$ the stiffness matrix. Moreover, damping effects are considered by a dissipative port with related variables $\boldsymbol{f}_{R, c}=-\overline{\boldsymbol{D}}_{c} \boldsymbol{e}_{R, c}$ with $\boldsymbol{f}_{R, c}$ the dissipative wrench applied on the bodies $i$ and $j$ expressed in the contact frame, $\boldsymbol{e}_{R, c}$ the corresponding twist, and $\bar{D}_{c}$ the damping coefficient matrix for the contact $c$. The overall Dirac structure for compliant contacts can be written as follows:

$$
\left[\begin{array}{c}
{ }^{0} \boldsymbol{W}_{c}^{i} \\
{ }^{0} \boldsymbol{W}_{c}^{j} \\
\boldsymbol{e}_{R, c} \\
\dot{\boldsymbol{s}}
\end{array}\right]=\left[\begin{array}{cc}
\mathbf{0}_{12 \times 12} & -\boldsymbol{A}_{t o t} \\
\boldsymbol{A}_{t o t}^{T} & \mathbf{0}_{12 \times 12}
\end{array}\right]\left[\begin{array}{c}
{ }^{0} \boldsymbol{T}_{i}^{0} \\
{ }^{0} \boldsymbol{T}_{j}^{0} \\
-\boldsymbol{f}_{R, c} \\
-\frac{\partial H_{s}}{\partial \boldsymbol{s}}
\end{array}\right]
$$

with $\boldsymbol{A}_{t o t}=\left[\begin{array}{ll}\boldsymbol{A} & -\boldsymbol{A} \\ \boldsymbol{A} & -\boldsymbol{A}\end{array}\right]^{T}$ and $\boldsymbol{A}$ a mapping of the form (3).

\section{E. Overall PH modeling}

In this section we finally formulate the PH equations of the whole system describing the joint object handling performed by a human and $m$ collaborative robots. Compliant contact is assumed for the connection of the object with the human and the first $k$ robots, while rigid contact is assumed for the rest of the connections, see Fig. 2 which details the interconnections between subsystems, clarifies the definition of each port variable used in each subsystem, and represents the port-based principle of the whole system.

The interconnection of all sub-systems results in a $\mathrm{PH}$ formulation of form (2) with state vector

$$
\begin{aligned}
\boldsymbol{x} & =\left[\boldsymbol{P}_{o}, \boldsymbol{X}_{o}, \boldsymbol{P}_{a}, \boldsymbol{Q}_{a}, \boldsymbol{S}_{a}\right]^{T}, \quad \text { and } \\
\boldsymbol{P}_{o} & =\left[\boldsymbol{p}_{o} ; \boldsymbol{l}_{o}\right]^{T}, \quad \boldsymbol{X}_{o}=\left[\boldsymbol{x}_{o}\right]^{T}, \quad \boldsymbol{P}_{a}=\left[\boldsymbol{p}_{h} ; \boldsymbol{p}_{1} ; \cdots ; \boldsymbol{p}_{m}\right]^{T}, \\
\boldsymbol{Q}_{a} & =\left[\boldsymbol{q}_{h} ; \boldsymbol{q}_{1} ; \cdots ; \boldsymbol{q}_{m}\right]^{T}, \quad \boldsymbol{S}_{a}=\left[\boldsymbol{s}_{h} ; \boldsymbol{s}_{1} ; \cdots ; \boldsymbol{s}_{k}\right]^{T}
\end{aligned}
$$

$\boldsymbol{J}(\boldsymbol{x})$ and $\boldsymbol{R}(\boldsymbol{x})$ matrices as reported in (11) and (12),

$$
\begin{aligned}
& \boldsymbol{J}=\left[\begin{array}{ccccc}
\mathbf{0} & -\tilde{\boldsymbol{I}} & \mathbf{0} & \mathbf{0} & -\boldsymbol{B} \\
\tilde{\boldsymbol{I}}^{T} & \mathbf{0} & \mathbf{0} & \mathbf{0} & \mathbf{0} \\
\mathbf{0} & \mathbf{0} & \mathbf{0} & -\boldsymbol{E} & -\boldsymbol{F} \\
\mathbf{0} & \mathbf{0} & \boldsymbol{E}^{T} & \mathbf{0} & \mathbf{0} \\
\boldsymbol{B}^{T} & \mathbf{0} & \boldsymbol{F}^{T} & \mathbf{0} & \mathbf{0}
\end{array}\right] \\
& \tilde{\boldsymbol{I}}=\left[\begin{array}{ll}
\boldsymbol{I}_{3} & \mathbf{0}_{3 \times 3}
\end{array}\right]^{T}, \\
& \boldsymbol{B}=\left[-z_{h} \boldsymbol{G}_{h}^{T} \boldsymbol{A}_{h}^{T},-z_{1} \boldsymbol{G}_{1}^{T} \boldsymbol{A}_{1}^{T}, \cdots,-z_{k} \boldsymbol{G}_{k}^{T} \boldsymbol{A}_{k}^{T}, 0, \cdots, 0\right], \\
& \boldsymbol{E}=\boldsymbol{I}_{\left(n_{h}+\sum_{r=1}^{m} n_{r}\right)} \\
& \boldsymbol{F}=\operatorname{diag}\left(-z_{h} \boldsymbol{J}_{h}^{T} \boldsymbol{A}_{h}^{T},-z_{1} \boldsymbol{J}_{1}^{T} \boldsymbol{A}_{1}^{T}, \cdots,-z_{k} \boldsymbol{J}_{k}^{T} \boldsymbol{A}_{k}^{T}\right), \\
& \boldsymbol{R}=\left[\begin{array}{ccccc}
z_{h} \boldsymbol{G}_{h}^{T} \boldsymbol{A}_{h}^{T} \overline{\boldsymbol{D}}_{h} \boldsymbol{A}_{h} \boldsymbol{G}_{h}+\sum_{r=1}^{k} z_{r} \boldsymbol{G}_{r}^{T} \boldsymbol{A}_{r}^{T} \overline{\boldsymbol{D}}_{r} \boldsymbol{A}_{r} \boldsymbol{G}_{r} & 0 & \boldsymbol{L} & 0 & 0 \\
\mathbf{0} & \mathbf{0} & \mathbf{0} & \mathbf{0} & \mathbf{0} \\
\boldsymbol{L}^{T} & \mathbf{0} & \boldsymbol{V} & \mathbf{0} & \mathbf{0} \\
\mathbf{0} & \mathbf{0} & \mathbf{0} & \mathbf{0} & \mathbf{0} \\
\mathbf{0} & \mathbf{0} & \mathbf{0} & \mathbf{0} & \mathbf{0}
\end{array}\right]
\end{aligned}
$$

mapping matrices (13) for control and interaction ports

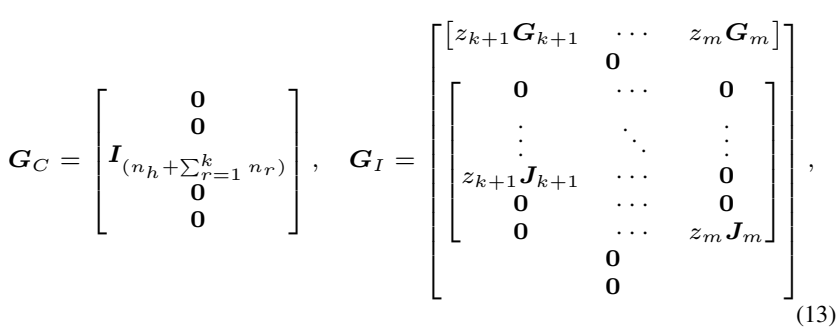

as well as $\boldsymbol{u}_{I}$ the wrenches acting on the interaction ports $\boldsymbol{u}_{I}=\left[\begin{array}{lll}{ }^{0} \boldsymbol{W}_{o, k+1}^{k+1} & \cdots & { }^{0} \boldsymbol{W}_{o, m}^{m}\end{array}\right]^{T}$, with $\boldsymbol{A}_{t}$ of form (3), $\boldsymbol{J}_{i}$ the Jacobians of the robots or human, $\overline{\boldsymbol{D}}_{t}$ the damping coefficient for the contact point $t$ and $\boldsymbol{D}_{i}$ the dissipation matrix for the robot(s) or human. The constraint for the rigid contact points can be described by: ${ }^{0} \boldsymbol{T}_{i}^{0}-{ }^{0} \boldsymbol{T}_{o, i}^{0}=\mathbf{0}_{6}$ for $i \in\{k+1, \cdots, m\}$.

Finally, changes in the interconnection of subsystems can be simply modelled by binary variables $z_{t}$ to manipulate e.g. the contact behavior of each sub-system, i.e. $z_{t}=$ Identity if the sub-system is connected to the rest of the system over contact point $t$ and $z_{t}=$ Zero if not.

\section{SAFETy-Enhancing ENERGY Shaping CONTROL}

The proposed model connects all sub-systems via power ports and thus, allows to easily install a flow-based energy monitoring system that observes energy flows between all sub-systems, especially the port connecting the human to the rest of the system. We propose safety metrics and a supervision-based controller to shape the energy exchanged with the human, whenever a harmful or fatiguing energy flow over the human/robot interaction (HRI) port is observed.

\section{A. Safety metrics for HRC}

In literature several metrics for the risk assessment of unintended and hazardous contacts between humans and robots exist. They mostly specify injury-related limits for mechanical hazards such as collisions. The most frequently investigated quantities in this context are transferred energy, force, and pressure observed at the collision points [24].

However, hardly any safety metrics related to continuous physical HRC are available in state-of-the-art literature. Inspired by the main injury-related factors known to be the total amount of discharged energy, the rate of discharge and the area over which energy is released, we defined the following safety principles to enhance user's safety during continuous physical collaboration with robots:

a. The maximum possible energy to be exchanged with the human and its rate of change should not violate a pre-defined safe threshold.

b. The pre-defined safe threshold should be adaptive based on user fatigue to decrease the risk of muscle injury.

Hazardous situations during HRC can be the consequence of unpredictable robot behavior resulting from e.g. false sensor readings or actuator failures. They may lead to a fast change of total energy or rate of change of energy exchanged with the human. Therefore, keeping the total energy in the 


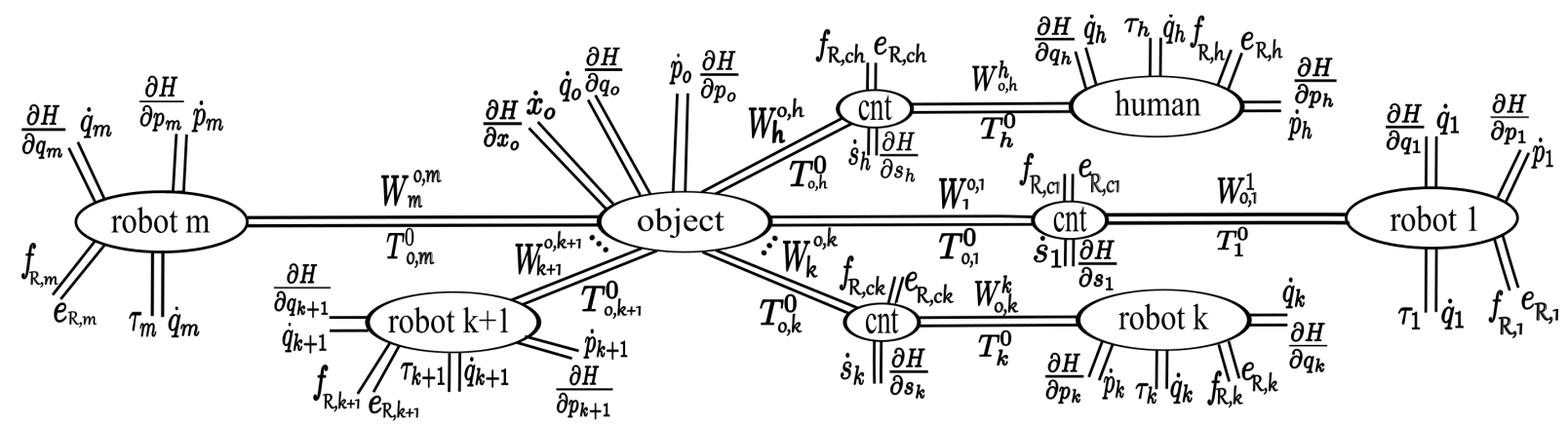

Fig. 2. Port-based representation of collaborative transportation of an object by a human and $m$ robots, each with either rigid contact (direct connection) or compliant contact (represented by (cnt)).

system below an acceptable limit $\left(H_{t o t}<H_{\max }\right)$ is a first step to improve safety. Such upper safety limits are the results of experimental energy-related injury analyses performed in literature, see [25], [26] for collision-based results on cranial bones and neck bones. Also the rate of change of energy, namely power, passing through the HRI port should be bounded $\left(P_{h}<P_{\max }\right)$. As currently ONLY metrics for safety evaluation during impacts are available, we use $P_{\max }$ found in impact studies, see e.g. [24], [27], while in future research we will attempt at deriving proper new thresholds for continuous interaction taking the specific human configuration into account.

Finally, human fatigue can be considered a further risk factor as muscle injuries may result from over-straining (see [28]-[31]). Therefore, we propose the aforementioned upper thresholds for energy and power to be not only functions of injury-related measures, but also of user fatigue.

\section{B. Control design}

The safety controller is represented as an independent $\mathrm{PH}$ system that operates on the basis of energetic information and energy flows in the system. The controller should interfere as little as possible with the execution of the task (e.g. transporting an object from an initial to a final position), while implementing the aforementioned safety principles without explicit knowledge of the human sub-system. Then the controller is designed based on the information provided from the port-based modeling of the system interacting with the human.

1) Nominal controller: As the coordination of multiple robots is beyond the scope of this paper and our aim is to illustrate the main principle of the proposed safety controller, without loss of generality we reduced the control problem to the tracking of pre-determined trajectories starting at an initial and ending at a final robot configuration. The desired equilibrium state for robot $r$ is considered $\left(\boldsymbol{x}_{r}, \boldsymbol{P}_{r}\right)=$ $\left(\boldsymbol{x}_{d e s, r}, \mathbf{0}\right)$, where $\boldsymbol{x}_{d e s, r}$ is the desired robot configuration. As controller we chose

$$
\boldsymbol{u}_{r}=\boldsymbol{K}_{p, r} \boldsymbol{x}_{e, r}+\boldsymbol{K}_{d, r} \dot{\boldsymbol{x}}_{e, r}
$$

with a global and unique minimum at the desired equilibrium and $\boldsymbol{x}_{e, r}=\boldsymbol{x}_{d e s, r}-\boldsymbol{x}_{r}$ the robot configuration error. The stiffness $\boldsymbol{K}_{p, r}$ connects the desired equilibrium and the current robot configuration, while the additional damping factor $\boldsymbol{K}_{d, r}$ helps stabilizing the controlled PH system.

2) Safety-enhancing adaptive controller: A safe system behavior during $\mathrm{HRC}$ is achieved by proper tuning of the parameters $\boldsymbol{K}_{p}$ and $\boldsymbol{K}_{d}$ of the nominal controller for all robots based on safety principles defined in Section IV-A.

The controlled system interacting with the human includes $m$ robots and the object with a total energy of

$$
H_{t o t}=\sum_{r=1}^{m} \frac{1}{2} \boldsymbol{x}_{e, r}^{T} \boldsymbol{K}_{p, r} \boldsymbol{x}_{e, r}+\sum_{r=1}^{m} H_{r}+H_{o}
$$

where $K_{p, r}$ is a diagonal matrix representing the stiffness factor in the controller of robot $r$, and $H_{r}$ and $H_{o}$ are the Hamiltonian functions of the robot $r$ and the object, respectively. Considering $H_{\max }$ to be the upper safe value of $H_{t o t}$, (15) can be re-written as follows:

$$
H_{e f f}=\boldsymbol{Q} \boldsymbol{K}_{p}=H_{\max }-\sum_{r=1}^{m} H_{r}-H_{o}
$$

where $\boldsymbol{Q}=\left[\boldsymbol{x}_{e, 1}^{T} \boldsymbol{x}_{e, 1}, \ldots, \boldsymbol{x}_{e, m}^{T} \boldsymbol{x}_{e, m}\right]$ and $\boldsymbol{K}_{p}=$ $\left[\boldsymbol{K}_{p, 1}, \ldots, \boldsymbol{K}_{p, m}\right]^{T}$. Thus, considering $H_{\max }$ to be the limit of the total energy, the stiffness factors for each robot controller are selected as follows:

$$
\boldsymbol{K}_{p}= \begin{cases}\boldsymbol{K}_{p} & H_{t o t} \leq H_{\max } \\ \boldsymbol{Q}^{\#} H_{e f f} & H_{t o t}>H_{\max }\end{cases}
$$

with $\boldsymbol{Q}^{\#}$ the Pseudoinverse of $\boldsymbol{Q}$. Please note that also a weighted Pseudoinverse could be used instead.

Next, the upper limit for the rate of change of the energy flow needs to be guaranteed. The power conservation property of the Dirac structure implies that the change in the stored energy of a system equals the sum of the power provided by the external ports and dissipative ports:

$$
\boldsymbol{e}_{R}^{T} \boldsymbol{f}_{R}+\boldsymbol{e}_{C}^{T} \boldsymbol{f}_{C}+\boldsymbol{e}_{I}^{T} \boldsymbol{f}_{I}=-\boldsymbol{e}_{S}^{T} \boldsymbol{f}_{S}=\dot{H}
$$

Applying this logic to the system, the total power transferred over the HRI port is written as:

$$
\begin{aligned}
P_{h} & =-\sum_{r=1}^{m} P_{c, r}+\sum_{r=1}^{m} \dot{H}_{r}+\dot{H}_{o}, \quad \text { with } \\
P_{c, r} & =\left(\boldsymbol{K}_{p, r} \boldsymbol{x}_{e, r}-\boldsymbol{K}_{d, r} \dot{\boldsymbol{x}}_{e, r}\right)^{T} \dot{\boldsymbol{x}}_{e, r}
\end{aligned}
$$


where $P_{c, r}$ is the power injected by the controller of robot $r$. Considering the maximum power applied to the human to be $P_{\max }$ we finally get

$$
P_{e f f}=\boldsymbol{V} \boldsymbol{K}_{d}=P_{m a x}+\sum_{r=1}^{m} \boldsymbol{x}_{e, i}^{T} \boldsymbol{K}_{p, r} \dot{\boldsymbol{x}}_{e, r}-\sum_{r=1}^{m} \dot{H}_{r}-\dot{H}_{o}
$$

with $\boldsymbol{V}=\left[\dot{\boldsymbol{x}}_{e, 1}^{T} \dot{\boldsymbol{x}}_{e, 1}, \ldots, \dot{\boldsymbol{x}}_{e, m}^{T} \dot{\boldsymbol{x}}_{e, m}\right]$ and $\boldsymbol{K}_{d}=$ $\left[\boldsymbol{K}_{d, 1}, \ldots, \boldsymbol{K}_{d, m}\right]^{T}$. In order to consider the upper and lower limits for power exchange between the human and the rest of the system, the sign of $P_{\max }$ is selected as follows

$$
P_{\max }= \begin{cases}-P_{\max } & P_{h} \leq-P_{\max } \\ P_{\max } & P_{h}>P_{\max }\end{cases}
$$

The damping factors for each robot controller are finally tuned to avoid unsafe rates of change of energy to be discharged over the HRI port, resulting in:

$$
\boldsymbol{K}_{d}= \begin{cases}\boldsymbol{K}_{d} & \left|P_{h}\right| \leq\left|P_{\max }\right|, \\ \boldsymbol{V}^{\#} P_{e f f} & \text { else }\end{cases}
$$

with $\boldsymbol{V}^{\#}$ the Pseudoinverse of $\boldsymbol{V}$.

Please note that the values of $\boldsymbol{K}_{p}$ will never become negative and thus, result in instability, as long as $H_{\max }$ is reasonably chosen. This can be easily checked by analyzing (17), where $Q$ is positive definite and thus, only a negative value of $H_{\text {eff }}$ can lead to a negative $\boldsymbol{K}_{p}$. However, $H_{\text {eff }}$ is computed based on (16), which will never become negative as long as $H_{\max }$, is larger than the two other terms in (16). This is always the case because a reduction of $H_{\text {eff }}$ would result in a reduction of $\boldsymbol{K}_{p}$, which again would reduce $\sum_{r=1}^{m} H_{r}$ and thus, would increase $H_{\text {eff }}$ again. A similar logic can also be applied for the adaptation of the $\boldsymbol{K}_{d}$ gain.

Overloading muscles may result in muscle pain, or even strain injury. Thus, apart from preventing dangerous robot behavior, we further aim at reducing the risk of muscle injury through adaptation of the power applied to the human based on estimation of the fatigue. Taking into account relations of fatigue and work, see [32], [33], a probability for human muscle fatigue is derived by integrating the energy flow over the HRI port,

$$
p_{\text {fatigue }}=\frac{\int P_{h} d t}{W_{h, \max }},
$$

with $W_{h, \max }$ the total performed work at which human muscle fatigue starts to be observed [32]-[34].

To take fatigue into account in the controller design, we control the energy flow over the HRI port by online adapting the value of $P_{\max }$ according to the level of muscle fatigue:

$$
P_{\max }=p_{\text {fatigue }} \tilde{P}_{\min }+\left(1-p_{\text {fatigue }}\right) \tilde{P}_{\max },
$$

with $\tilde{P}_{\min }$ and $\tilde{P}_{\max }$ being the minimum and, respectively, the maximum human contribution to the task.

\section{RESUlTS}

We present simulation results for the port-Hamiltonian model of the considered HRC task, including also the proposed energy-based safety controller.

\section{A. Simulation of the HRC model}

Validation of the modeling approach was performed on a simple test scenario considering the collaborative object manipulation by a 2 DOF serial robot manipulator with revolute joints and a 2 DOF human arm with shoulder and elbow joints moving in the vertical plane. The total mass and inertia tensor of the object were considered to be $M_{o}=5 \mathrm{~kg}$ and $I_{o}=\operatorname{diag}(0.006,0.04,0.04) \quad\left[\mathrm{kgm}^{2}\right]$ respectively, while the dynamic and geometric parameters of the robot were assumed as follows: link lengths $l_{1, r}=l_{2, r}=1 \mathrm{~m}$, mass and inertia of the segments $m_{1, r}=m_{2, r}=1 \mathrm{~kg}$ and $I_{1, r}=I_{2, r}=0.084 \mathrm{kgm}^{2}$. The friction in the joints was neglected for the sake of simplicity. For the human arm the following values were assumed for simulation: $m_{1, h}=$ $1.4, m_{2, h}=1.1 \mathrm{~kg}, l_{1, h}=0.3, l_{2, h}=0.33 \mathrm{~m}$, and $I_{1, h}=$ $0.025, \quad I_{2, h}=0.045 \mathrm{kgm}^{2}$ and $\boldsymbol{K}_{p, h}=\operatorname{diag}(100,100)$ $[\mathrm{N} / \mathrm{m}], \boldsymbol{K}_{d, h}=\operatorname{diag}(10,10)[\mathrm{N} \cdot \mathrm{s} / \mathrm{m}]$.

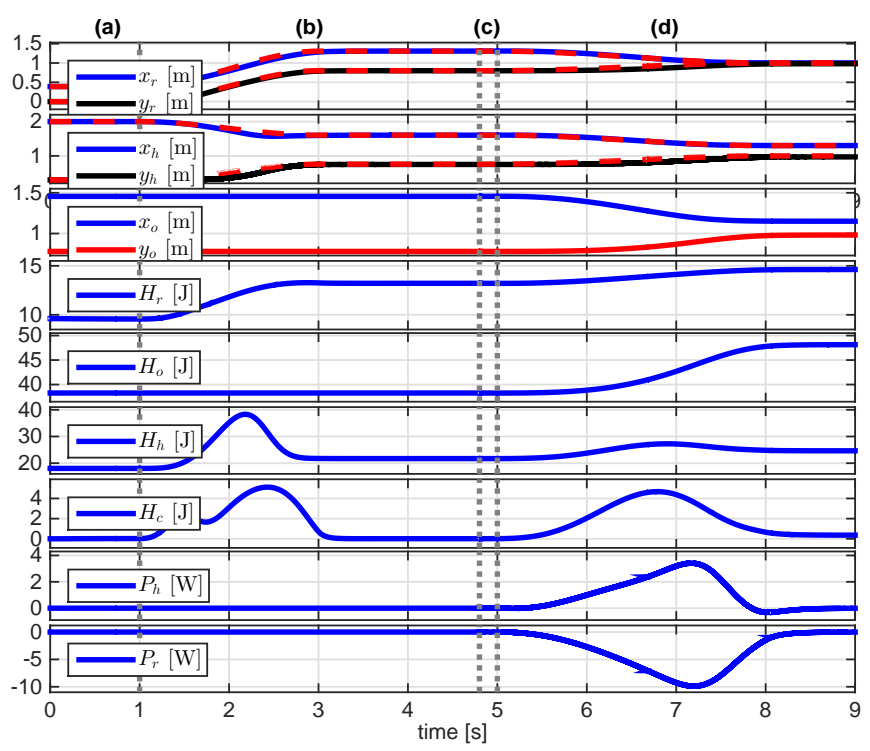

Fig. 3. Evaluation of the energy-based HRC model. In the first two top plots, dashed red lines show the reference positions and solid lines show obtained values. The four phases (a), (b), (c) and (d) are separated by dotted gray lines.

Figure 3 illustrates the energetic behavior of each subsystem during the experiment. Four different phases were considered: (a) non-contact initial condition from initial to time equal to $1 \mathrm{~s}$, (b) approaching the object between $1 \mathrm{~s}$ and $4.8 \mathrm{~s}$ and, (c) establishing contact at $4.8 \mathrm{~s}$ and keeping the current configuration to $5 s$, (d) transporting the object to the desired position from $5 s$ to the end. Constant stiffness and damping factors $\boldsymbol{K}_{p}=\operatorname{diag}(2000,2000)[\mathrm{N} / \mathrm{m}]$ and $\boldsymbol{K}_{d}=\operatorname{diag}(100,100)[\mathrm{N} \cdot \mathrm{s} / / \mathrm{m}]$ were considered. In phase (c) contact between human-object and robot-object is established by simply activating the corresponding contact variables. The three top plots show a good trajectory tracking performance since the reference positions, which are defined for human and robot independently, are well followed using the aforementioned controller. The Hamiltonian of each subsystem, as well as the power exchanged between them, is 
monitored thanks to the port-based modeling. For example, the transferred powers $P_{h}$ and $P_{r}$ are zero before establishing connection, while their value after connection to the object represents their injected or absorbed power during task execution. The value of the power exchanged over the robotobject interaction point $\left(P_{r}\right)$ is almost three times bigger than the power exchanged over the human-object interaction port $\left(P_{r}\right)$ and thus, indicates a larger contribution of the robot than the human to the execution of the task. Please consider that the positive value for the human power corresponds to the power transferred from the object to the human. The initial change in the Hamiltonian of the robot and human during phase (b) is due to movements towards the obstacle, while further changes during phase (d) are because of transporting the object. Finally, slow gradual changes in the Hamiltonian of the object can be explained by changes of its potential energy since it was transferred to a higher position.

\section{B. Validation of the safety-enhancing control approach}

A trajectory tracking task was considered, and the following two scenarios were studied to validate the safetyenhancing controller design proposed in previous sections. In the first scenario, we evaluate the robot behavior while satisfying aforementioned safety criteria, and in the second scenario we focus on the adaptation of the robot actions based on the estimated human fatigue.

1) Scenario I: The human and the robot transport the object from an initial to a final configuration, while the robot avoids a dynamic obstacle placed very close to the path and suddenly appearing at time $0.8 \mathrm{~s}$ to $1.3 \mathrm{~s}^{2}$ The system performance for cases with and without safety controller is depicted in Fig. 4 with $H_{\max }=65 \mathrm{~J}, P_{\max }=15 \mathrm{~W}$, $\boldsymbol{K}_{p}=\operatorname{diag}(1000,1000)[\mathrm{N} / \mathrm{m}]$ and $\boldsymbol{K}_{d}=\operatorname{diag}(100,100)$ $[\mathrm{N} \cdot \mathrm{s} / \mathrm{m}]$. Looking at the results for the case without safety controller, it can be seen that the robot reaction to avoid collision with the obstacle results in violating constraints for the maximum power to be allowed to be exchanged with the human, i.e. $P_{h}>P_{\max }$ as well as a potential harmful increase of the total energy of the system interacting with the human, i.e. $H_{t o t}>H_{\max }$. The two top plots in Fig. 4 show that the actual trajectory minimally deviates from the desired one as a consequence of the safety controller adjusting its parameters $\boldsymbol{K}_{p}$ and $\boldsymbol{K}_{d}$ to prevent violation of safety constraints.

2) Scenario II: A repetitive task of object transportation is studied, which allows to observe an increasing level of human fatigue. We considered $P_{\text {high }}=8.3 \mathrm{~W}$ and $P_{\text {low }}=$ $5 \mathrm{~W}$. As can be observed in Fig. 5, the control parameters are adjusted automatically to prevent extra power applied to the human if the estimated user fatigue increases. In both cases, with and without safety controller, the system tracks well the desired trajectory as depicted in the two top plots. Moreover, with increasing user fatigue, the maximum allowed power

\footnotetext{
${ }^{2}$ Please note that collision avoidance during HRC is out of focus of this paper and the case of a suddenly appearing obstacle is used as representative example of many other unpredictable and hazardous situations that can happen during HRC.
}

to be transferred over the human-object interaction port is reduced, which results in an increase of $\boldsymbol{K}_{d}$ to prevent the violation of safety constraints. For illustration purposes, a relatively low value for $W_{h, \max }=1200 \mathrm{~J}$ was considered, which resulted in a rather fast increase of fatigue.

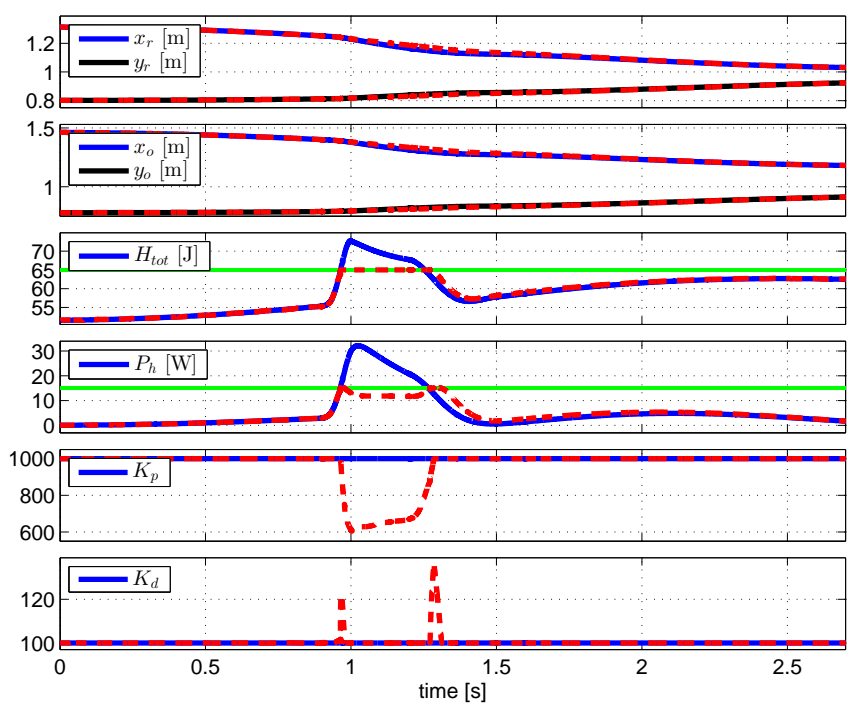

Fig. 4. Validation of energy-based adaptive shared controller. Solid blue and black lines show signals using nominal controller, dashed red lines show signals using safety-enhancing adaptive controller and green lines in the two middle plots show the considered safety thresholds.
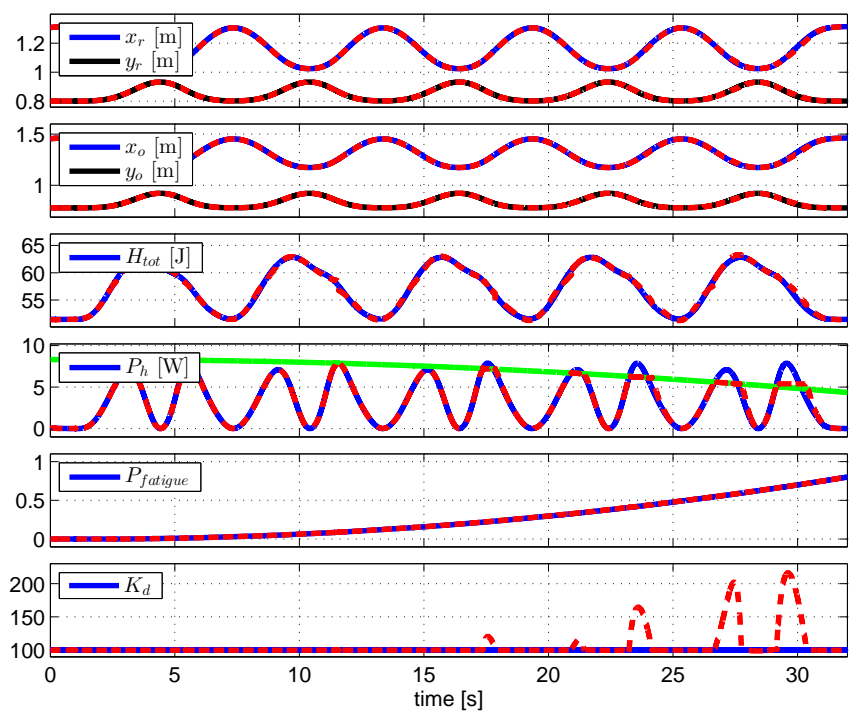

Fig. 5. Validation of energy-based adaptive shared controller for user fatigue reduction. Solid blue and black lines show signals using nominal controller, dashed red lines show signals using the safety-enhancing adaptive controller and green lines in the fourth plot shows the considered adaptive safety threshold.

\section{CONCLUSIONS}

Collaborative tasks where a human-robot team jointly manipulates bulky objects can be suitably described using bond graphs and energy flows through ports to cover the 
different, continuous and time-varying contact situations that arise in such scenarios. The formalism of port-Hamiltonian systems was adopted here to model each sub-system (robots, humans, objects) and their interconnection. We designed a monitoring system that observes energy flows between sub-systems, with a compliance controller that shapes these flows so as to enhance human safety. Metrics for safety considered the maximum allowed energy to be exchanged with humans and its maximum rate of change, along with measures that take human fatigue into account. A complete stability analysis of the controlled system is available, but omitted here due to space constraints.

While limiting the energy of the robots and the power transferred to the human enhances the safety of the system, eliminating the possibility of injuries in HRC requires further consideration of other factors such as the constrained or unconstrained state of the human body. Moreover, we are currently experimenting the safety-enhancing approach in different HRC tasks, where reliable dynamic models of robots and objects are needed. In particular, an on-line identification scheme of object dynamics would be more appropriate than using a priori information. Nonetheless, the approach relies on power and energy flows and we only need to monitor and bound such physical quantities, rather than canceling dynamic terms by control. In this sense, our controller is intrinsically more robust to uncertainties. Other safety metrics that consider the continuous nature of tasks and the actual configuration of bodies are also being evaluated.

\section{REFERENCES}

[1] A. Albu-Schäffer, O. Eiberger, M. Grebenstein, S. Haddadin, C. Ott, T. Wimbock, S. Wolf, and G. Hirzinger, "Soft robotics," IEEE Robotics \& Automation Mag., vol. 15, no. 3, pp. 20-30, 2008.

[2] A. Albu-Schäffer, S. Haddadin, C. Ott, A. Stemmer, T. Wimböck, and G. Hirzinger, "The DLR lightweight robot: Design and control concepts for robots in human environments," Industrial Robot, vol. 34, no. 5, pp. 376-385, 2007.

[3] A. Bicchi and G. Tonietti, "Fast and soft-arm tactics," IEEE Robotics \& Automation Mag., vol. 11, no. 2, pp. 22-33, 2004.

[4] Y. Yamada, Y. Hirasawa, S. Huang, and Y. Umetani, "Fail-safe human/robot contact in the safety space," in Proc. 5th IEEE Int. Work. on Robot and Human Communication, 1996, pp. 59-64.

[5] S. Haddadin, A. Albu-Schäffer, A. De Luca, and G. Hirzinger, "Collision detection and reaction: A contribution to safe physical humanrobot interaction," in Proc. IEEE/RSJ Int. Conf. on Intelligent Robots and Systems, 2008, pp. 3356-3363.

[6] A. De Luca and F. Flacco, "Integrated control for pHRI: Collision avoidance, detection, reaction and collaboration," in Proc. 4th IEEE Int. Conf. on Biomedical Robotics and Biomechatronics, 2012, pp. 288-295.

[7] M. Geravand, F. Flacco, and A. De Luca, "Human-robot physical interaction and collaboration using an industrial robot with a closed control architecture," in Proc. IEEE Int. Conf. on Robotics and Automation, 2013, pp. 4000-4007.

[8] S. Haddadin, Towards Safe Robots: Approaching Asimov's 1st Law. Springer, 2013.

[9] M. Geravand and A. Peer, "Safety constrained motion control of mobility assistive robots," in Proc. 5th IEEE Int. Conf. on Biomedical Robotics and Biomechatronics, 2014, pp. 1073-1078.

[10] T. Tadele, T. de Vries, and S. Stramigioli, "Combining energy and power based safety metrics in controller design for domestic robots," in Proc. IEEE Int. Conf. on Robotics and Automation, 2014.
[11] M. Laffranchi, N. Tsagarakis, and D. Caldwell, "Improving safety of human-robot interaction through energy regulation control and passive compliant design," in Human Machine Interaction - Getting Closer, M. Inaki, Ed. InTech, 2012, ch. 8.

[12] B. Maschke, A. van der Schaft, and P. Breedveld, "An intrinsic Hamiltonian formulation of the dynamics of LC-circuits," IEEE Trans. on Circuits and Systems I: Fundamental Theory and Applications, vol. 42, no. 2, 1995.

[13] D. Eberard, L. Lefevre, and B. Maschke, "Multiscale coupling in heterogeneous diffusion processes: A port-based approach," in Proc. Int. Conf. on Physics and Control, 2005, pp. 543-547.

[14] A. Macchelli, C. Melchiorri, and S. Stramigioli, "Port-based modeling and simulation of mechanical systems with rigid and flexible links," IEEE Trans. on Robotics, vol. 25, no. 5, pp. 1016-1029, 2009.

[15] M. Ishikawa, A. Neki, J. Imura, and S. Hara, "Energy preserving control of a hopping robot based on hybrid port-controlled Hamiltonian modeling," in Proc. IEEE Conf. on Control Applications, 2003, pp. $1136-1141$.

[16] A. Mersha, R. Carloni, and S. Stramigioli, "Port-based modeling and control of underactuated aerial vehicles," in Proc. IEEE Int. Conf. on Robotics and Automation, 2011, pp. 14-19.

[17] F. Ficuciello, R. Carloni, L. Visser, and S. Stramigioli, "PortHamiltonian modeling for soft-finger manipulation," in Proc. IEEE/RSJ Int. Conf. on Intelligent Robots and Systems, 2010, pp. $4281-4286$

[18] V. Duindam and S. Stramigioli, Modeling and control for efficient bipedal walking robots. Springer, 2009.

[19] V. Duindam, A. Macchelli, S. Stramigioli, and H. Bruyninckx, Modeling and control of complex physical systems. Springer, 2009.

[20] A. Macchelli, "Port Hamiltonian systems. A unified approach for modeling and control finite and infinite dimensional physical systems," Ph.D. dissertation, DEIS, University of Bologna, 2003.

[21] C. Secchi, S. Stramigioli, and C. Fantuzzi, Control of interactive robotic interfaces: A Port-Hamiltonian approach, ser. STAR. Springer, 2007, vol. 29.

[22] N. Diolaiti, C. Melchiorri, and S. Stramigioli, "Contact impedance estimation for robotic systems," IEEE Trans. on Robotics, vol. 21, no. 5, pp. 925-935, 2005.

[23] V. Duindam and S. Stramigioli, "Modeling the kinematics and dynamics of compliant contact," in Proc. IEEE Int. Conf. on Robotics and Automation, vol. 3, 2003, pp. 4029-4034.

[24] R. Behrens and N. Elkmann, "Study on meaningful and verified thresholds for minimizing the consequences of human-robot collisions," in Proc. IEEE Int. Conf. on Robotics and Automation, 2014, pp. 33783383.

[25] J. Wood, "Dynamic response of human cranial bone," J. of Biomechanics, vol. 4, no. 1, pp. 1-12, 1971.

[26] N. Yoganandan, F. Pintar, D. Maiman, J. Cusick, A. Sances Jr, and P. Walsh, "Human head-neck biomechanics under axial tension," Medical Engineering \& Physics, vol. 18, no. 4, pp. 289-294, 1996.

[27] "BG/BGIA risk assessment recommendations according to machinery directive: Design of workplaces with collaborative robots," Deutsche Gesetzliche Unfallversicherung, Tech. Rep. U001/2009e, 2011.

[28] S. Dugan and W. Frontera, "Muscle fatigue and muscle injury," Physical Medicine and Rehabilitation Clinics of North America, vol. 11, no. 2 , pp. 385-403, 2000.

[29] A. Genaidy et al., "Biomechanical tolerance limits for manual lifting tasks: A tool to control back injuries," in Proc. Int. Conf. of IEEE Engineering in Medicine and Biology Soc., 1989, pp. 803-805.

[30] K. Veiersted, R. Westgaard, and P. Andersen, "Pattern of muscle activity during stereotyped work and its relation to muscle pain," International Arch. of Occupational and Environmental Health, 1990.

[31] S. Mair, A. Seaber, R. Glisson, W. Garrett, et al., "The role of fatigue in susceptibility to acute muscle strain injury," American J. of Sports Medicine, vol. 24, no. 2, pp. 137-143, 1996.

[32] M. Hagberg, "Work load and fatigue in repetitive arm elevations," Ergonomics, vol. 24, no. 7, pp. 543-555, 1981.

[33] T. Moritani, A. Nagata, H. deVries, and M. Muro, "Critical power as a measure of physical work capacity and anaerobic threshold," Ergonomics, vol. 24, no. 5, pp. 339-350, 1981.

[34] T. Sakurai, M. Toda, S. Sakurazawa, J. Akita, K. Kondo, and Y. Nakamura, "Detection of muscle fatigue by the surface electromyogram and its application," in 9th IEEE/ACIS Int. Conf. on Computer and Information Science, 2010, pp. 43-47. 